\title{
Depuis 2013, le bac S est inadapté aux études supérieures de physique et de chimie
}

\begin{abstract}
Atterrés par ce constat alarmant, les présidents de I'Union des Professeurs de classes préparatoires Scientifiques aux grandes écoles (UPS), de I'Union des Professeurs de Physique et de Chimie (UdPPC) et de la Société Française de Physique (SFP) se sont adressés à Madame la ministre de l'Éducation nationale pour faire connaître l'ampleur du diagnostic* lors d'une entrevue, accordée le 25 mars 2015.
\end{abstract}

Le bac S (scientifique) est inadapté pour aborder les cinq années d'enseignement supérieur, universités et grandes écoles, ouvrant aux carrières scientifiques, celles qui mènent aux métiers de l'ingénieur comme celles qui préparent à la recherche.

En 2014, parmi les 163100 lauréats du bac S, seuls environ 50000 jeunes, le tiers, se lancent dans des études supérieures scientifiques pour obtenir un diplôme de sortie bac+5. (Le bac $S$, comme les autres bacs généraux, a un taux de réussite important, $89 \%$ en 2006 et $92 \%$ en 2014 .)

L'enseignement supérieur a ainsi accueilli à la rentrée de septembre 2013 des étudiants ayant suivi les nouveaux programmes du lycée. La SFP, I'UdPPC et I'UPS ont alors décidé à la fin de I'année scolaire 2013-2014 de consulter* les enseignants de première année de l'enseignement supérieur (universités, IUT, classes préparatoires).

Constat : mener une démonstration développée en plusieurs étapes, maitriser les outils de base de mathématiques, et s'astreindre à la rigueur pour construire un raisonnement sont des objectifs éliminés de la formation donnée aux bacheliers de la série $S$.

Éloignée de ces valeurs, une séquence de champs scientifiques, sans lien, sans objectif de progression, est proposée, limitée aux vertus culturelles, certes importantes, mais totalement inadaptées à la constitution d'un corpus de pratiques scientifiques. Une réduction des horaires, diverse dans le détail, mais globalement d'une heure en première et terminale, à la fois pour les mathématiques et la physique-chimie, associée à une évolution conséquente du programme de mathématiques, mine les bases de la formation scientifique au lycée, si nécessaire à l'économie française pour reconquérir un socle industriel actuellement très affaibli. Seuls les travaux personnels encadrés (TPE) échappent à la critique. En classe de première, proposer aux lycéens de préparer un dossier synthétisant leur travail de recherche sur un sujet choisi ou de fabriquer un montage mécanique ou électronique recueille une opinion générale positive à laquelle nous nous associons.

Loin d'améliorer la transition lycée-postbac des étudiants des filières scientifiques, la réforme a creusé le fossé.

- L'enquête montre que les meilleurs étudiants scientifiques ont tendance à se détourner de la physique. Ils quittent le lycée avec une vision erronée des sciences physique et chimie. De nombreux étudiants sont désarmés et déçus quand ils constatent leur incapacité à utiliser des outils formels et à pratiquer des démarches scientifiques rigoureuses.

- Ces programmes ont été conçus avec l'idée que la majorité (2/3) des élèves de filière $S$ ne poursuivra pas d'études supérieures scientifiques. Cela se traduit par un renoncement à en asseoir les bases indispensables. Ce repliement sur un vernis culturel superficiel asséné par des arguments d'autorité est bien loin d'une vraie culture, tant les fondements d'une véritable démarche scientifique en sont absents. Les bacheliers $\mathrm{S}$ collectionnent des connaissances cloisonnées non consolidées : cette "culture scientifique mal digérée » survole de manière superficielle quelques sujets "scientifiques », bannit toute vision d'un continuum interdisciplinaire et ne construit aucun ancrage pour une véritable formation.

- La compréhension des démarches scientifiques est une construction lente, qui ne saurait attendre l'entrée dans I'enseignement supérieur. Elle requiert, en amont, une pratique concrète de la démarche de modélisation mathématique, essentielle à l'enseignement même et aux disciplines que sont la physique et la chimie. Ces concepts, ces outils sont absolument indispensables aux 50000 étudiants formés dans les filières scientifiques de I'enseignement supérieur, en France ou à l'étranger.

Il est urgent d'agir, car l'effondrement durable de l'enseignement des sciences, physique et chimie, au lycée aura des conséquences graves, non seulement sur la formation de nos futurs citoyens mais aussi sur la formation et le recrutement des cadres scientifiques (techniciens, ingénieurs, entrepreneurs, chercheurs, professeurs...) dont la France a besoin pour maintenir son rang au sein d'une économie mondialisée.

Sylvie Bonnet, présidente de l'UPS Alain Fontaine, président de la SFP

* Pour l'UdPPC, deux publications en 2013 et 2014 Vincent Parbelle, président de l'UdPPC www.udppc.asso.fr/bupdoc/consultation/article-bup.php?ID_fiche $=21693$
www.udppc.asso.fr/bupdoc/consultation/article-bup.php?ID_fiche $=21402$ Pour la SFP

www.sfpnet.fr/uploads/tinymce/ResultatsenqueteSFPprogrammelycee-1.pdf 